\title{
Knockdown of Novel IncRNA TCONS_00028652 in Zebrafish Affects Embryonic Vasculature Development
}

\author{
Chune Zhou, Shuqiang Zhang, Junguo Ma and Xiaoyu Li* \\ College of Life Science, Henan Normal University, Xinxiang, Henan 453007, P. R. China \\ *For correspondence: lixiaoyu65@263.net; 041106@htu.edu.cn \\ Received 28 October 2020; Accepted 06 January 2021; Published 25 March 2021
}

\begin{abstract}
Long non-coding RNAs (lncRNA) are increasingly being regard as potential key regulators of biological process, however, little is known about the function of most of them. The involvement of a novel lncRNA (ENSDART000000150571), previously named TCONS_00028652, in intersegmental vessel development was investigated in this study. TCONS_O0028652, having a single exon (568 bp), is located in chromosome 16 at position from 15786804 to 15786237 , which was previously identified as an embryonic and adult heart-enriched lncRNA. Bioinformatics analysis and annotation of TCONS_00028652 was performed using online databases. Its protein-coding potential was assessed using online softwares: the Coding Potential Assessing Tool (CPAT) and the Coding Potential Calculator (CPC). To verify its real existence, a cloned fragment was proliferated by designing primers against 5' and 3' exon-flanking sequence. Subsequently, spatiotemporal expression during zebrafish embryonic development was determined using real-time quantitative PCR (qPCR) and whole mount in situ hybridization. We found that lncRNA TCONS_00028652 was generally expressed throughout early stages of zebrafish embryonic development and predominantly in embryonic brain, tail, and heart. Knockdown of TCONS_00028652 using morpholino oligonucleotides (MO) resulted in intersegmental vessel defects, suggesting that TCONS_00028652 is indispensable for embryonic vascular development in zebrafish. Using Tg (flil:EGFP) transgenic fish expressing a cardiovascular marker gene, loss of function experiments confirmed that TCONS_O0028652 was involved in embryonic intersegmental vessel development. Our results may lead to valuable understanding of lncRNAs functions in zebrafish embryonic development and molecular mechanisms of embryonic cardiovascular development. (C) 2021 Friends Science Publishers
\end{abstract}

Keywords: IncRNA TCONS_00028652; Zebrafish; Embryonic development; Spatiotemporal expression; Intersegmental vessel Abbreviations: qPCR: quantitative real-time polymerase chain reaction; LncRNA: long noncoding RNA; Se: the primary intersegmental vessel; MO: morpholino oligonucleotide; Tg: transgenic; ANOVA: analysis of variance; DIG: digoxigenin; hpf: hours post-fertilization; CRISPR: clustered regularly interspaced short palindromic repeats; EGFP: enhanced green fluorescent protein; ESCs: embryonic stem cells.

\section{Introduction}

Recent applications of high-throughput sequencing technology have uncovered that less than $2 \%$ of the human genome encodes proteins (Esteller 2011; ENCODE Project Consortium 2012; Kellis et al. 2014) and most of the remaining genome that does not encode protein is made up of so called non-coding RNA (Mattick and Makunin 2006; Mercer et al. 2009; Adams et al. 2017). On the basis of mature transcript size, less than 200 nucleotide can be defined as small RNAs, greater than 200 nucleotide can be named as long ncRNAs (lncRNAs) and another type of ncRNA is housekeeping small RNAs, referred as regulatory RNAs (e.g., rRNA and tRNA). Amongst small RNAs, microRNAs (miRNAs) are the best studied. However, as the largest number of ncRNAs (Chowdhury et al. 2013), lncRNAs have attracted recent attention for the diverse gene regulation activity they provide at transcriptional, posttranscriptional, and epigenetic levels (Gutschner and Diederichs 2012). Hitherto, a large number of lncRNAs have been identified in animals, but their functions remain largely undefined (Atkinson et al. 2012; Derrien et al. 2012; Batista and Chang 2013).

It is necessary for embryonic development and adult survival to have normal cardiovascular system. In zebrafish, the cardiovascular system is developed before other organ systems during embryogenesis, with circulation beginning as early as $24 \mathrm{~h}$ post fertilization (hpf) (Fishman and Chien 1997). A functional vascular system is indispensable for supplying nutrients, hormones, immune cells, and oxygen for developing tissues and organs, as well as for eliminating toxic metabolic waste products. Cardiovascular development is a finely regulated process that necessitated abundant of genes (Kathiriya et al. 2015). 
Recent studies have indicated that IncRNAs play key roles in cardiovascular development (Rayner and Liu 2016). Several studies revealed that lncRNAs have a key role in cardiovascular development.

Zebrafish has become as popular animal in many area of research for its more rapid development speed, relatively shorter breeding cycle, and easier genetic manipulation (Vesterlund et al. 2011). In particular, they are an exceptional model animal for studying vascular development because they not only have transparent embryos and can be manipulated genetically (Bradbury 2004), but also their embryos can survive without blood circulation for approximately 7 days post fertilization. Thus, it is easy to discern vascular mutants. Transgenic zebrafish expressing enhanced green fluorescent protein (EGFP) $[T g$ (flk:EFGP), $T g$ (fli1:EGFP)] throughout their vasculature greatly facilitate in vivo studies of vessel formation (Lawson and Weinstein 2002; Jin et al. 2005). In this study, the expression pattern and zebrafish embryonic development function of TCONS_00028652 were explored.

\section{Materal and Methods}

\section{Animal husbandry}

Zebrafish (Danio rerio) were obtained from the Institute of Hydrobiology, Chinese Academy of Science (Wuhan, China). The transgenic $\mathrm{Tg}$ (flil:EGFP) (Friend leukemia virus integration 1) zebrafish line was from the Animal Center, Qixiu Campus of Nantong University, China. Embryos obtained from natural spawning of wild-type zebrafish were incubated and maintained at a light period of $14 / 10 \mathrm{~h}$ (light/dark) in accordance with described procedures (Westerfield 1995) and they were staged as previous report (Kimmel et al. 1995). All animal experiments were approved by the Institutional Animal Care and Use Committee at Henan Normal University. Embryos from wild-type $\mathrm{AB}$ strains were used for expression analysis, and transgenic strains [Tg (flil: GEFP)] were used for functional analyses. Two male and one female fish were collected in mating cages the night before embryo collection. The embryos were staged according to hours post-fertilization (hpf). The developmental stages selected for this study were $0,2,6,12,24,48,60$ and 72 hpf. For clearing whole embryos to observe gene expression, melanin pigment production was disrupted by raising embryos in 0.003\% 1-phenl-2-thiourea (P3755; Sigma, St Louis, USA) before $24 \mathrm{hpf}$.

\section{The identification and cloning of TCONS_00028652}

We annotated TCONS_00028652 using bioinformatics databases, such as Ensemble genome browser 98 (http://asia.ensembl.org/index.html), lncRNAdb (http://www.lncrnadb.rog/), and the UCSC genome browser (http://genome.ucsc.edu/). TCONS_00028652
(ENSDART00000150571) located in chromosome 16 at position from 15786804 to 15786237 and has only an exon (568 bp). TCONS_00028652 (ENSDART00000150571) is a transcript of non-coding gene si: dkeyl11b14.2 (ENSDARG00000096145). Cloned fragment was amplified, by designing primers against 5' and 3' exonflanking sequences, and then sequenced by GENEWIZ biotechnology Co. Ltd. (Jiangsu, China). The cloned sequence was aligned with the si: dkeyl11b14.2 sequence using NCBI blast in order to confirm the correct sequence. Then, we assessed its protein-coding potential using the online softwares: the Coding Potential Assessing Tool (CPAT) and the Coding Potential Calculator (CPC).

\section{Quantitative real time PCR (qPCR)}

Embryos gathered at various developmental stages were stored at $-80^{\circ} \mathrm{C}$ until they were processed. Total RNA was extracted from 50 embryos at each stage using RNAiso Plus (TaKaRa Biotechnology Co., Ltd. China). With a Nanodrop-2000, total RNA content was calculated from absorbance at $260 \mathrm{~nm}$ and RNA purity was verified by the $\mathrm{A}_{260} / \mathrm{A}_{280}$ ratio (> 1.8).

First strand cDNA was reversely transcribed from 1-5 $\mu \mathrm{g}$ total RNA with a HIFIScript 1 st Strand cDNA Synthesis kit (Cwbiotich, China) used in accordance with manufacturer's instructions. Relative lncRNA expression levels were measured with qPCR and $\beta$-actin was used as the internal reference. The qPCR conditions were: polymerase activation for $10 \mathrm{~min}$ at $95^{\circ} \mathrm{C}$ and 40 cycles of $95^{\circ} \mathrm{C}$ for $10 \mathrm{~s}$ and $54-60^{\circ} \mathrm{C}$ for $30 \mathrm{~s}$. The data were calculated using the $2^{-\Delta \Delta \mathrm{Ct}}$ method. All primers were synthesized by GENEWIZ Biological Company (Suzhou, China) and are shown in Table 1.

\section{Whole mount in Situ hybridization}

In order to investigate the spatiotemporal expression pattern of TCONS_00028652, in situ hybridization with whole mount embryos was performed. Embryos were fixed in $4 \%$ paraformaldehyde. The TCONS_00028652 PCR fragment was amplified from cDNA (Cwbiotich, China) and cloned into pGEM-T easy plasmid (Promega, USA). The construct was linearized with ApaI or NsiI. Digoxigenin-labeled antisense RNA probes were transcribed in vitro with Sp6 or T7 RNA polymerases using a DIG RNA labeling kit (Roche, Germany). In situ hybridization with zebrafish embryos at different stages was performed in accordance with the standard detection method (Thisse and Thisse 2007).

\section{Morpholino injection}

To investigate the function of TCONS_00028652 in zebrafish embryonic vasculature development, we analyzed effects of TCONS_00028652 loss of function on zebrafish embryonic development. Loss of function was created by 
injecting antisense morpholino oligonucleotides (MO) into one-cell zygotes. One tactics would be to inject MOs targeted against lncRNA splice sites in an attempt to disrupt maturation. The other tactics would be to inject MOs designed against highly conserved regions supposed to be important to play its roles (Ulitsky et al. 2011). However, TCONS_00028652 is a single exon lncRNA, thus targeting an IncRNA splice site was infeasible. Therefore, we designed MO with Gene Tools to target a highly conserved region of TCONS_00028652. The MO sequence was 5'GCTTTTTTGATAACTCACCATGCCG-3', close to the 3' UTR. An equal volume of standard morpholino oligomers was used as a control. Morpholinos were dissolved in milliQ water as $1 \mathrm{~m} M$ stock solutions and diluted to $300 \mu M$ working solutions. For transgenic ( $\mathrm{Tg}$ : flil-GEFP) strains, 200 one-cell stage embryos were injected with about $5 \mathrm{~nL}$ of morpholino solution with a FemotoJet (Eppendorf). Zebrafish strain ( $\mathrm{Tg}$ : flil-EGFP) carries an endothelialspecific EGFP reporter that can visualize the details of developing blood vessels in vivo. Meanwhile, an equal number of one-cell-stage embryos were injected by standard morpholino oligomers, which do not specifically target genes in zebrafish embryos. Morphants were evaluated at 3 dpf with fluorescence microscopy.

\section{Statistical analysis}

The qPCR experiment was carried out in triplicate. Data are described as means \pm SD. Comparisons between control and test were evaluated by one-way analysis of variance (ANOVA) using S.P.S.S. 17.0. Probability $(P)<0.05$ was deemed as statistically significant.

\section{Results}

\section{Identification of A novel IncRNA TCONS_00028652}

A previous research identified TCONS_00028652 as an embryo and adult heart enriched lncRNA (Wang et al. 2017), but its function is elucidate. In order to study its function, its real existence is firstly verified through proliferating a cloned fragment using cDNA of TCONS_00028652 as template by designing primers against 5' and 3' exon-flanking sequences (Fig. 1 c). The length of cloned fragment was 1092 bp (Fig. 1d). The results of sequencing and alignment confirmed its real existence. A lack of protein-coding potential was assessed using two online software: CPAT and CPC analysis (Fig. $1 \mathrm{a}, \mathrm{b})$. The same result was obtained using the NCBI ORF finder tool, which further confirmed that it does not encode protein.

Expression profile of LncRNA TCONS_00028652 at different stages of zebrafish embryonic development

To clarify the expression pattern of TCONS_00028652 during early zebrafish embryogenesis, we performed qPCR with embryos at eight developmental stages $(2,6,12,24$, $36,48,60$, and $72 \mathrm{hpf}$ ). The results showed that TCONS_00028652 was expressed throughout embryonic development (Fig. 2i), with the highest expression at 6 and $12 \mathrm{hpf}$. The expression profile in zebrafish embryo indicates that TCONS_00028652 may participate in zebrafish embryogenesis.

To some extent, gene function can be predicted by studying gene expression distribution. In order to confirm the spatiotemporal expression pattern of TCONS_00028652 in embryos at different developmental stages, we executed whole mount in situ hybridization of TCONS_00028652 using a digoxigenin-labeled antisense RNA. The in situ hybridization indicated that TCONS_00028652 was highly expressed from fertilization through $72 \mathrm{hpf}$, highly consistent with the qPCR profile. This result suggests that TCONS_00028652 may have a crucial role throughout embryonic development (Fig. 2). The early appearance of TCONS_00028652 (0 hpf) raises the possibility of maternal origin or regulation.

In early stages (0, 2, and $10 \mathrm{hpf})$, TCONS_00028652 expression was diffuse (Fig. $2 \mathrm{a}-\mathrm{c}$ ). However, in later stages $(24,36,48,60$ and $72 \mathrm{hpf})$, it was expressed predominantly in brain and tail (Fig. 2d-h). Additionally, TCONS_00028652 was expressed in other organs and tissues, such as muscle, heart, neural tissues, tail fin, and pectoral tissues, suggesting that TCONS_00028652 might play multiple functions during zebrafish embryogenesis.

\section{Morpholino knockdown of LncRNA TCONS_00028652 and knockdown efficiency validation}

In order to explore the potential role of TCONS_00028652 in vasculature development, we created TCONS_00028652 loss of function by injecting $\mathrm{MO}$, an oligonucleotide targeting a conserved region of TCONS_00028652 (Fig. 3e), into one- to two-cell stage $T g$ (flil:EGFP) transgenic zebrafish embryos. As a control, "standard morpholino" was injected, which does not target any zebrafish genes. The promoter for Flil (Friend leukemia virus integration 1), is specifically expressed in hematopoietic and endothelial cells (Melet et al. 1996a), was applied to activate enhanced green fluorescent protein (EGFP) expression in all blood vessels throughout embryogenesis (Lawson and Weinstein 2002).

We tested the efficiency of synthesized TCONS_00028652-MO using qPCR and whole mount in situ hybridization. The results declared that TCONS_00028652 expression was significantly reduced in TCONS_00028652-MO morphants compared with noninjected (NI) embryos and control-MO embryos at $48 \mathrm{hpf}$ (Fig. 3).

\section{Abnormal vascular and heart phenotypes in LncRNA TCONS_00028652-MO embryos}


Table 1: Primers used for $\mathrm{qPCR}$ and in situ hybridization in this study

\begin{tabular}{ll}
\hline Primers & Sequences \\
\hline TCONS_00028652-qPCR-F & GACACGGAAAGGATTGACAG \\
TCONS_00028652-qPCR-R & TTCGTTATCGGAATGAACCAG \\
TCONS_00028652-Probe-F & GAATACCGCAGCTAGGAA \\
TCONS_00028652-Probe-R & CGTTATCGGAATGAACCA \\
Fli1-Probe-F & TCGTCCCCGCAGACCC \\
Fli1-Probe-R & GACGCTGGGATTGGGGTAAA \\
\hline
\end{tabular}

\section{$\mathrm{F}$, forward, R, reverse}
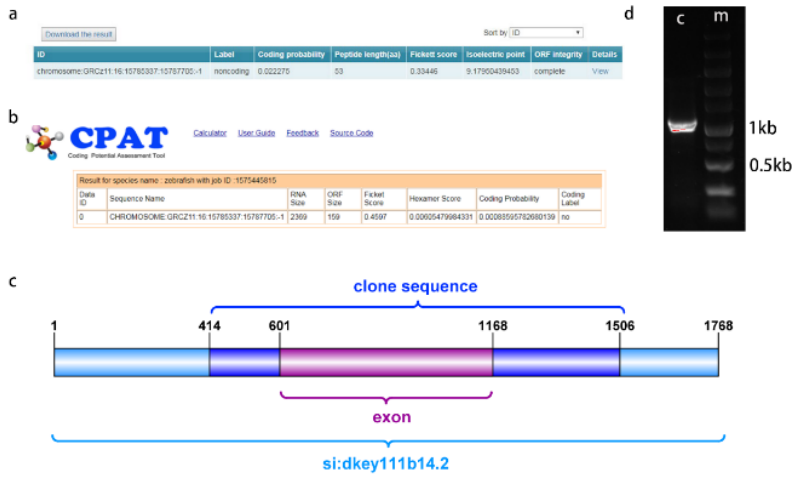

Fig. 1: Identification of TCONS_00028652

a: The protein-coding analysis using CPC; $\mathbf{b}$ : The protein-coding analysis using CPAT; $\mathbf{c}$ : The genetic structure diagram of TCONS_00028652; $\mathbf{d}$ : The agarose gel image of clone fragment; $\mathrm{C}$ : clone M: marker
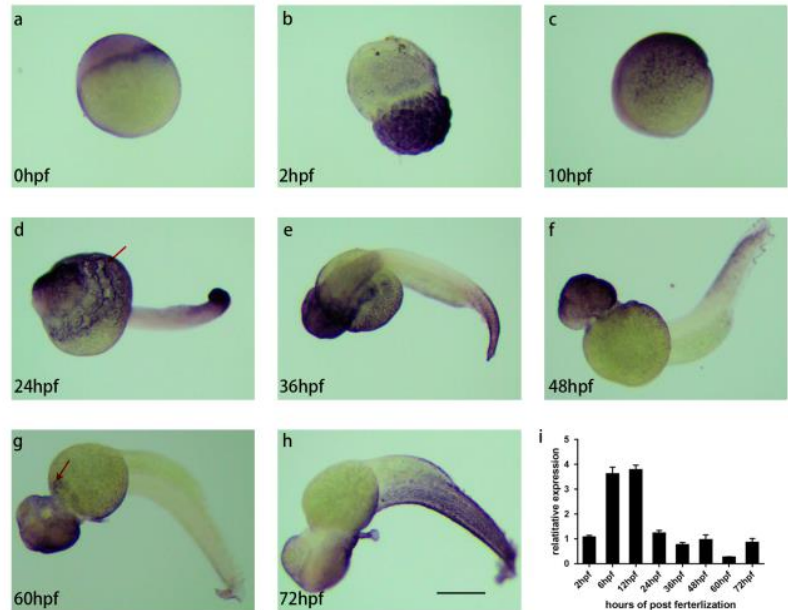

Fig. 2: Expression of TCONS_00028652 during embryonic development in zebrafish

Scale bar $=50 \mu \mathrm{m}$; $\mathbf{a}-\mathbf{h}:$ in situ hybridiazation showing TCONS_00028652 expression in zebrafish embryos at different stages of development $(0,2,10,24,36,48,60$, and $72 \mathrm{hpf}) ;$ i: The relative expression of TCONS_00028652 in zebrafish embryos at different stages of development; Red arrow points to the heart

Cardiac edema was observed in TCONS_00028652-MO morphants (Fig. 4f) compared with NI embryos and CONMO embryos (Fig. 4e, g). Further, TCONS_00028652-MO embryos had significantly reduced body length (Fig. 4h). Previous reports have proposed a dynamic connection

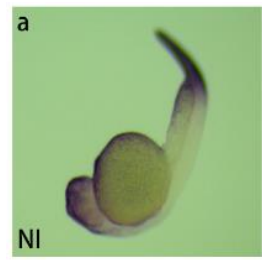

d
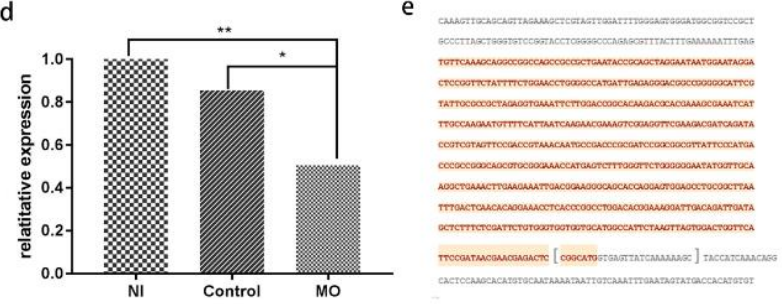

Fig. 3: Morpholino knockdown of TCONS_00028652 in early zebrafish embryonic development

Scale bar $=50 \mu \mathrm{m}$; a-c: In situ hybridization showing the expression of TCONS 00028652 from the TCONS_00028652-MO knockdown embryos or controlMO embryos (48 hpf); d: Relative expression level of TCONS_00028652; e: The target sequence showed by the bracket
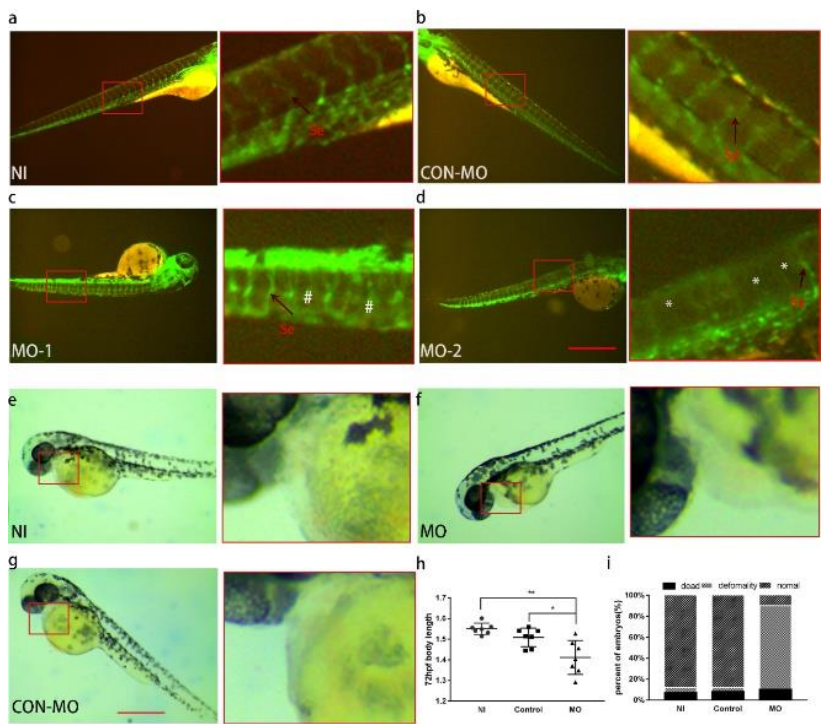

Fig. 4: Effect of TCONS_00028652 knockdown on vasculature development and heart morphology in $T g$ (fli1: EGFP) transgenic zebrafish embryos

Scale bar $=50 \mu \mathrm{m}$; a-d: Fluorescent images of $T g$ (fli1: EGFP) at $3 \mathrm{dpf}$; \#: points to the interruption of Se; *: points to the absent of vascular structures; The arrow points to the Se; e-g: The heart morphology in TCONS_00028652-MO embryos comparted compared to the NI and CON embryos; i: The quantity of the rate of deformities; NI: Non-injection; CON: Control-MO; Se: Intersegmental vessel

between somitogenesis and vasculature development (Torres-Vazquez et al. 2004; Mei et al. 2010). Thus, in order to determine if TCONS_00028652 is essential for vasculature development, transgenic $\mathrm{Tg}$ (flil:GEFP) embryos were injected with morpholino against TCONS_00028652, and vasculature development was evaluated. As shown in Fig. 4, compared with normal vasculature in NI embryos (Fig. 4a) and CON-MO embryos (Fig. 4b), the primary intersegmental vessel (Se) failed to 
sprout and form in TCONS_00028652-MO embryos. Moreover, vascular structures were absent or abnormal (Fig. 4d) and extensive defects were observed in TCONS_00028652-MO embryos at $3 \mathrm{dpf}$ (Fig. 4c). A higher rate of deformity was observed in TCONS_00028652-MO embryos (Fig. 4i). This result strongly indicates that TCONS_00028652 knockdown can disturb intersegmental vessel development in zebrafish.

\section{Fli1 expression in IncRNA TCONS_00028652-MO embryos with in Situ hybridization}

To further verify the effect of TCONS_00028652 knockdown on fish embryonic vascular development, whole-mount in situ hybridization was conducted in fish embryos, with endothelial cell marker flil used as a monitor. The result showed that Se sprouts could be clearly observed in NI embryos (Fig. 5a) or CON-MO embryos (Fig. 5b); however, in TCONS_00028652-MO embryos, the corresponding vascular structure was absent or abnormal (Fig. 5c, d). Expression of fill in IncRNA TCONS_00028652-MO embryos indicated missing endothelial cells (Fig. 5), which is consistent with the aberrant EGFP expression.

\section{Discussion}

In recent decades, with the employment of high-throughput deep sequencing approaches, the majority of long noncoding RNAs have been identified in many species, including zebrafish. Over 3000 lncRNAs have been identified in zebrafish embryos and adult tissues (Ulitsky et al. 2011; Pauli et al. 2012; Kaushik et al. 2013; Wang et al. 2017). However, the functions of most lncRNAs remain unclear.

Several reports now describe the function and molecular regulatory mechanism of lncRNAs in human diseases, especially cancer. However, evidence for lncRNAs function in animal development is still lacking. Deciphering the functional roles and regulatory mechanisms of development-related IncRNAs can reveal molecular mechanisms of diseases and also expand avenues for creating disease therapies. Recently, several developmentrelated IncRNAs have been identified and characterized in zebrafish and mouse (Dinger et al. 2008; Pauli et al. 2015; Luo et al. 2016) and the results suggest that lncRNAs can substantially affect gene regulation during embryogenesis. For instance, lncRNA tie-AS was found to be involved in transcriptional regulation of vascular development (Li et al. 2009; Chowdhury et al. 2018). LncRNA braveheart was required for cardiovascular lineage commitment and activated the cardiac vascular gene network (Klattenhoff $e t$ al. 2013; Hou et al. 2017). LncRNA fendrr showed tissuespecific expression and was essential for proper heart and body wall development in mouse (Grote et al. 2013). Three other cardiovascular-related lncRNAs, TERMINATOR, $A L I E N$ and PUNISHER, specifically expressed in
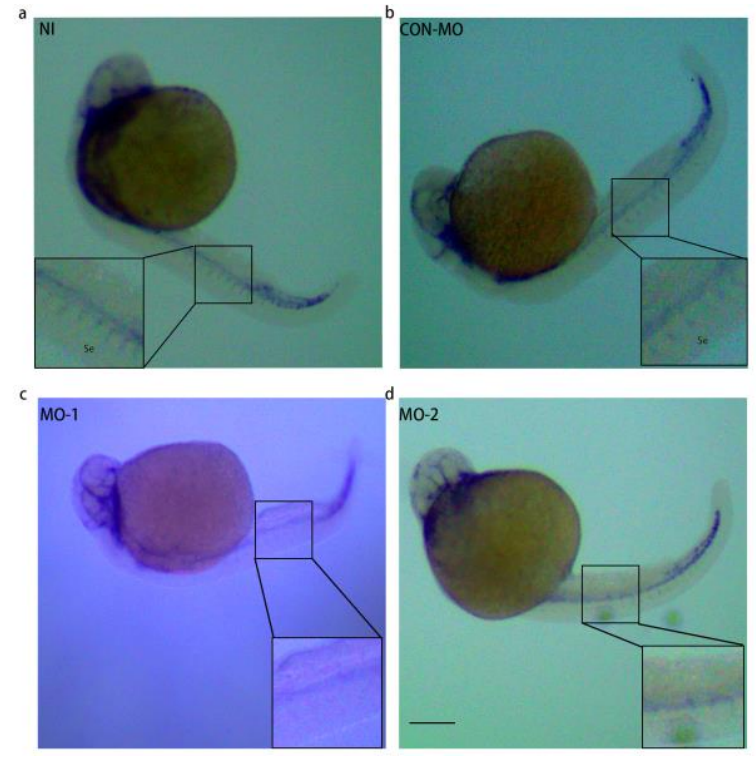

Fig. 5: Fill expression in TCNONS_O0028652 -MO embryos as shown by in situ hybridization

Scale bar $=50 \mu \mathrm{m}$; $\mathbf{a}$ : NI embryos; $\mathbf{b}$ : CON embryos; $\mathbf{c}$ and $\mathbf{d}$ : TCONS_00028652MO embryos; Black arrow points to the Se; NI: Non-injection; CON: Control-MO; Se: Intersegmental vessel

undifferentiated pluripotent stem cells, cardiovascular progenitors, and differentiated endothelial cells, respectively, hence suggesting involvement in vertebrate cardiovascular development (Kurian et al. 2015). A novel lncRNA durga arising from the first exon of Kalirin, played a key role in axonal development, nerve growth and synaptic re-modeling, was reported to modulate dendrite density and kalirin expression in zebrafish (Sarangdhar et al. 2017). The sequence and central nervous system-restricted expression of lincRNA TUNA are strikingly conserved in vertebrates. Accordingly, TUNA knockdown in zebrafish impaired locomotor function, which suggests that lincRNA TUNA plays a vital role in pluripotency and neural differentiation in embryonic stem cells and is associated with adult vertebrate neurological function (Lin et al. 2014). In our study, lncRNA TCONS_00028652 was generally expressed in early stages of embryonic development in zebrafish, predominantly in the embryonic brain, tail and heart. Knockdown of TCONS_00028652 resulted in intersegmental vessel defects, suggesting that TCONS_O0028652 is indispensable for zebrafish embryonic vasculature development.

One effective strategy for pursuing gene function is to disrupt gene expression by gene knockdown or knockout. Gene knockout technology, such as CRISPR/Cas9, which is a widely used loss of gene function method, can alter the DNA gene locus. Alternatively, knockdown methods using morpholino oligonucleotides, a preferred zebrafish knockdown reagent made of 25 nucleotides substituting a morpholine ring and non-ionic phosphorodiamidate linkages for the ribose ring and phosphodiester backbone found in DNA and RNA (Mathew et al. 2019), can be used to 
investigate lncRNAs (Li et al. 2009; Ulitsky et al. 2011; Goudarzi et al. 2019). MOs interfere with gene function by targeting either splice sites, in an attempt to disrupt lncRNA maturation, or highly conserved regions, presumed to be functional sites. MOs targeting splice sites or highly conserved regions in lncRNAs Cyrano and Megamind caused similar developmental defects (Ulitsky et al. 2011). In the present investigation of TCONS_00028652, only a highly conserved region could be targeted by MO because TCONS_00028652 has only one exon and hence no splice sites. Our results showed that the knockdown by $\mathrm{MO}$ targeting the highly conserved region of TCONS_00028652 caused developmental phenotype defects in embryonic blood vessels, as observed in Tg (flil:EGFP) transgenic fish expressing a cardiovascular marker gene.

Zebrafish are commonly considered a powerful model for studying genes and proteins regulating embryonic vascular development (Goishi and Klagsbrun 2004; McKinney and Weinstein 2008). A large amount of established techniques enable us to easily research zebrafish vessel development from early endothelial cell differentiation through adult vessel morphology (McKinney and Weinstein 2008). Transgenic zebrafish with GFP expression driven by the zebrafish flil promoter can be used to clearly visualize vasculature formation during zebrafish embryogenesis. Flil is known as an endothelial cell marker in mouse (Melet et al. 1996b) and is also expressed during vascular development in zebrafish embryos (Thompson et al. 1998). In this study, transgenic zebrafish (flil:GEFP) facilitated the visualization of intersegment vessel defects induced by MO injection, proving their vascular-specific utility. Our results showed that embryonic heart and blood vessel defects could be observed clearly in TCONS_00028652 morphants expressing the cardiovascular marker gene flil.

\section{Conclusion}

The results of qPCR and whole mount in situ hybridization showed that IncRNA TCONS_00028652 is generally expressed throughout early stages of embryonic development, predominantly in the embryonic brain, tail, and heart in zebrafish. Moreover, knockdown of TCONS_00028652, using morpholino oligonucleotides, resulted in intersegmental vessel defects, suggesting that TCONS_00028652 is indispensable for zebrafish embryonic vascular development. However, the regulatory mechanism details of TCONS_00028652 in embryonic intersegmental vessel development will further explore in future studies.

\section{Acknowledgements}

This research was supported by the Key Research Project of Henan University in China (Grant No.19zx011) and the $5^{\text {th }}$ Science \& Technology Innovation Fund of Henan, China (5101044100023).

\section{Author Contributions}

ZCE conceived the research and performed all experiments in this study. ZSQ provided transgenic zebrafish and valuable technical advice. MJG gave technical assistance. LXY provided research instruction throughout the study and reviewed the manuscript.

\section{Conflict of Interest}

There is no conflict of interest among the authors

\section{Data Availability Declaration}

All data, reported in this article are available with the corresponding authors and can be provided upon request

\section{Ethics Approval}

The guidelines for research on animals were duly observed

\section{References}

Adams BD, C Parsons, L Walker, WC Zhang, FJ Slack (2017). Targeting noncoding RNAs in disease. J Clin Invest 127:761-771

Atkinson S, S Marguerat, J Bahler (2012). Exploring long non-coding RNAs through sepuencing. Semin Cell Dev Biol 23:200-205

Batista P, H Chang (2013). Long noncoding RNAs: Cellular address codes in development and disease. Cell 152:200-205

Bradbury J (2004). Small fish, big science. PLoS Biol 2; Article e148

Chowdhury D, YE Choi, ME Brault (2013). Charity begins at home: Noncoding RNA functions in DNA repair. Nat Rev Mol Cell Biol 14:181-189

Chowdhury TA, C Koceja, S Eisa-Beygi, BP Kleinstiver, SN Kumar, CW Lin, K Li, S Prabhudesai, JK Joung, R Ramchandran (2018). Temporal and spatial post-transcriptional regulation of zebrafish tiel mRNA by long noncoding RNA during brain vascular assembly. Throm Vasc Biol 38:1562-1575

Derrien T, R Guigó, JR Roderic (2012). The long non-coding RNAs: A new (p)layer in the "dark matter". Front Genet 2; Article 107

Dinger ME, PP Amaral, TR Mercer, KC Pang, SJ Bruce, BB Gardiner, ME Askarian-Amiri, K Ru, G Solda, C Simons, SM Sunkin, ML Crowe, SM Grimmond, AC Perkins, JS Mattick (2008). Long noncoding RNAs in mouse embryonic stem cell pluripotency and differentiation. Genomics Res 18:1433-1445

ENCODE Project Consortium (2012). An integrated encyclopedia of DNA elements in the human genome. Nature 489:57-74

Esteller M (2011). Non-coding RNAs in human disease. Nat Rev Genet 12:861-874

Fishman M, K Chien (1997). Fashioning the vertebrate heart: Earliest embryonic decisions. Development 124:2099-2177

Goishi K, M Klagsbrun (2004). Vascular endothelial growth factor and its receptors in embryonic zebrafish blood vessel development. Curr Top Dev Biol 62:127-152

Goudarzi M, K Berg, LM Pieper, AF Schier (2019). Individual long noncoding RNAs have no overt functions in zebrafish embryogenesis, viability and fertility. Elife 8; Article e40815

Grote P, L Wittler, D Hendrix, F Koch, S Wahrisch, A Beisaw, K Macura, G Blass, M Kellis, M Werber, BG Herrmann (2013). The tissue-specific lncRNA Fendrr is an essential regulator of heart and body wall development in the mouse. Dev Cell 24:206-214

Gutschner T, S Diederichs (2012). The hallmarks of cancer: A long noncoding RNA point of view. RNA Biol 9:703-719 


\section{Novel lncRNA TCONS_00028652 in Zebrafish / Intl J Agric Biol, Vol 25, No 4, 2021}

Hou J, H Long, C Zhou, S Zheng, H Wu, T Guo, Q Wu, T Zhong, T Wang (2017). Long noncoding RNA braveheart promotes cardiogenic differentiation of mesenchymal stem cells in vitro. Stem Cell Res Ther 8:4-16

Jin SW, D Beis, T Mitchell, JN Chen, DY Stainier (2005). Cellular and molecular analyses of vascular tube and lumen formation in zebrafish. Development 132:5199-5209

Kathiriya IS, EP Nora, BG Bruneau (2015). Investigating the transcriptional control of cardiovascular development. Circulat Res 110:700-714

Kaushik K, VE Leonard, S Kv, MK Lalwani, S Jalali, A Patowary, A Joshi, VScaria, S Sivasubbu (2013). Dynamic expression of long non-coding RNAs (lncRNAs) in adult zebrafish. PLoS One 8; Article e83616

Kellis M, B Wold, M Snyder, B Bernstein, A Kundaje, G Marinov, L Ward, E Birney, G Crawford, J Dekker, I Dunham, L Elnitski, P Farnham, E Feingold, M Gerstin, M Giddings, D Gilbert, T Gingeras, E Green, R Guigo, T Hubbard, J Kent, J Lieb, R Myers, M Pazin, B Ren, J Stamatoyannopoulos, Z Weng, K White, R Hardison (2014). Defining functional DNA elements in the human genome. Proc Natl Acad Sci USA 111:6131-6138

Kimmel C, WKSR Ballard, B Ullmann, T Schilling (1995). Stages of Embryonic Development of the Zebrafish. Dev Dyn 203:1238-1246

Klattenhoff CA, JC Scheuermann, LE Surface, RK Bradley, PA Fields, ML Steinhauser, H Ding, VL Butty, L Torrey, S Haas, R Abo, M Tabebordbar, RT Lee, CB Burge, LA Boyer (2013). Braveheart, a long noncoding RNA required for cardiovascular lineage commitment. Cell 152:570-583

Kurian L, A Aguirre, I Sancho-Martinez, C Benner, T Hishida, TB Nguyen, P Reddy, E Nivet, MN Krause, DA Nelles, CR Esteban, JM Campistol, GW Yeo, JC Izpisua Belmonte (2015). Identification of novel long noncoding RNAs underlying vertebrate cardiovascular development. Circulation 131:1278-1290

Lawson ND, BM Weinstein (2002). In vivo imaging of embryonic vascular development using transgenic zebrafish. Dev Biol 248:307-318

Li K, Y Blum, A Verma, Z Liu, K Pramanik, NR Leigh, CZ Chun, GV Samant, B Zhao, MK Garnaas, MA Horswill, SA Stanhope, PE North, RQ Miao, GA Wilkinson, M Affolter, R Ramchandran (2009). A noncoding antisense RNA in tie-1 locus regulates tie-1 function in vivo. Blood 115:133-139

Lin N, KY Chang, Z Li, K Gates, ZA Rana, J Dang, D Zhang, T Han, CS Yang, TJ Cunningham, SR Head, G Duester, PD Dong, TM Rana (2014). An evolutionarily conserved long noncoding RNA TUNA controls pluripotency and neural lineage commitment. Mol Cell 53:1005-1019

Luo S, JY Lu, L Liu, Y Yin, C Chen, X Han, B Wu, R Xu, W Liu, P Yan, W Shao, Z Lu, H Li, J Na, F Tang, J Wang, YE Zhang, X Shen (2016). Divergent lncRNAs regulate gene expression and lineage differentiation in pluripotent cells. Cell Stem Cell 18:637-652

Mathew S, A Sivadas, P Sehgal, K Kaushik, SK Vellarikkal, V Scaria, S Sivasubbu (2019). Methods to study long noncoding RNA expression and dynamics in zebrafish using RNA sequencing. Meth Mol Biol 1912:77-110

Mattick JS, IV Makunin (2006). Non-coding RNA. Hum Mol Genet 15:17-19
McKinney MC, BM Weinstein (2008). Using the zebrafish to study vessel formation. Meth Enzymol 444:65-97

Mei J, S Liu, Z Li, JF Gui (2010). Mtmr8 is essential for vasculature development in zebrafish embryos. BMC Dev Biol 10:96-106

Melet FMB, DJ Rossi, L Zhang (1996a). Generation of a novel Fli-1 protein by gene targeting leads to a defect in thymus development and a delay in Friend virus-induced erythroleukemia. Mol Cell Biol 16:2708-2718

Melet F, BRDJ Motro, L Zhang, A Bernstein (1996b). Generation of a novel Fli-1 protein by gene targeting leads to a defect in thymus development and a delay in Friend virus-induced erythroleukemia. Mol Cell Biol 6:2708-2718

Mercer TR, ME Dinger, JS Mattick (2009). Long non-coding RNAs: Insights into functions. Nat Rev Genet 10:155-159

Pauli A, TG Montague, KA Lennox, MA Behlke, AF Schier (2015). Antisense oligonucleotide-mediated transcript knockdown in zebrafish. PLoS One 10; Article e0139504

Pauli A, E Valen, MF Lin, M Garber, NL Vastenhouw, JZ Levin, L Fan, A Sandelin, JL Rinn, A Regev, AF Schier (2012). Systematic identification of long noncoding RNAs expressed during zebrafish embryogenesis. Genome Res 22:577-591

Rayner KJ, PP Liu (2016). Long noncoding RNAs in the heart: The regulatory roadmap of Cardiovascular development and diseases. Circulat Cardiovasc Genet 9:101-103

Sarangdhar MA, D Chaubey, A Bhatt, M Km, M Kumar, S Ranjan, B Pillai (2017). A novel long non-coding RNA, durga modulates dendrite density and expression of kalirin in zebrafish. Front Mol Neurosci 10; Article 95

Thisse C, B Thisse (2007). High-resolution in situ hybridization to wholemount zebrafish embryos. Nat Protoc 3:59-69

Thompson MA, DG Ransom, SJ Pratt, H MacLennan, MW Kieran, HW Detrich, B Vail, TL Huber, B Paw, AJ Brownlie, AC Oates, A Fritz, MA Gates, A Amores, N Bahary, WS Talbot, H Her, DR Beier, JH Postlethwait, LI Zon (1998). The cloche and spadetail genes differentially affect hematopoiesis and vasculogenesis. Dev Biol 197:248-269

Torres-Vazquez J, AD Gitler, SD Fraser, JD Berk, NP Van, MC Fishman, S Childs, JA Epstein, BM Weinstein (2004). Semaphorin-plexin signaling guides patterning of the developing vasculature. Dev Cell 7:117-123

Ulitsky I, A Shkumatava, CH Jan, H Sive, DP Bartel (2011). Conserved function of lincRNAs in vertebrate embryonic development despite rapid sequence evolution. Cell 147:1537-1550

Vesterlund L, H Jiao, P Unneberg, O Hovatta, J Kere (2011). The zebrafish transcriptome during early development. BMC Dev Biol 11; Article 30

Wang L, X Ma, X Xu, Y Zhang (2017). Systematic identification and characterization of cardiac long intergenic noncoding RNAs in zebrafish. Sci Rep 7; Article 1250

Westerfield M (1995). The zebrafish book: A guide for the Laboratory Use of the Zebrafish (Danio rerio). University of Oregon Press, Eugene Oregon, USA 